\title{
On the gravitational signature of zonal flows in Jupiter-like planets: An analytical solution and its numerical validation
}

\author{
Dali Kong $^{\text {a }}$, Keke Zhang ${ }^{\text {b,1 }}$, and Gerald Schubert ${ }^{\mathrm{c}}$ \\ ${ }^{a}$ Key Laboratory of Planetary Sciences, Shanghai Astronomical Observatory, \\ Chinese Academy of Sciences, Shanghai 200030, China, and Center for \\ Geophysical and Astrophysical Fluid Dynamic, University of Exeter, Exeter, EX4 \\ $4 Q E, U K$ \\ ${ }^{\mathrm{b}}$ Center for Geophysical and Astrophysical Fluid Dynamic, University of Exeter, \\ Exeter, EX4 4QE, UK, and Lunar and Planetary Science Laboratory, Macau \\ University of Science and Technology, Macau \\ ${ }^{\mathrm{c}}$ Department of Earth, Planetary and Space Sciences, University of California, Los \\ Angeles, CA 90095-1567, USA
}

\begin{abstract}
In late 2016, the Juno spacecraft will provide an accurate spectrum of the Jovian zonal gravitational coefficients that would be affected by both the deep zonal flow, if it exists, and the basic rotational distortion. We derive the first analytical solution, under the spheroidal-shape approximation, for the density anomaly induced by an internal zonal flow in rapidly rotating Jupiter-like planets. We compare the density anomaly of the analytical solution to that obtained from a fully numerical solution based on a three-dimensional finite element method; the two show excellent agreement. We apply the analytical solution to a rapidly rotating Jupiter-like planet and show that there exists a close relationship between the spatial structure of the zonal flow and the spectrum of zonal gravitational coefficients. We check the accuracy of the spheroidal-shape approximation by computing both the spheroidal and non-spheroidal solutions with exactly the same physical parameters. We also discuss implications of the new analytical solution for interpreting the future high-precision gravitational measurements of the Juno spacecraft.
\end{abstract}

Key words: Gaseous Planets, Zonal Flow, Rotation,

$\overline{1}$ Corresponding author. E-mail address: kzhang@ex.ac.uk 


\section{Introduction}

The Juno spacecraft, which has just arrived at Jupiter, might measure the gravitational signature of a zonal flow possibly taking place in the planet's deep interior. Although progress has been made in modeling the Jovian zonal winds (for example, Busse, 1976; Heimpel et al., 2005; Jones and Kuzanyan, 2012; Gastine and Wicht, 2012), achieving the realistic physical parameters will probably never be possible and extrapolating the solutions from a numerically accessible model over many orders of magnitude is unreliable. If a theoretical relationship can be established between the zonal flow and the zonal-flow-induced gravitational field, however, high-precision gravitational measurements expected from the Juno mission might provide a "direct" way of probing the interior zonal flow.

The gravitational potential $V_{g}$ in the exterior of a rotationally distorted Jupiterlike planet can be expanded in terms of Legendre functions $P_{n}$,

$$
V_{g}(r, \theta)=-\frac{G M}{r}\left[1-\sum_{n=2}^{\infty} J_{n}\left(\frac{R_{e}}{r}\right)^{n} P_{n}(\cos \theta)\right], r \geq R_{e}
$$

where $M$ is the mass of the planet, $n$ takes even integers, $(r, \theta, \phi)$ are spherical polar coordinates with $\theta=0$ at the axis of rotation, $G=6.67384 \times$ $10^{-11} \mathrm{~m}^{3} \mathrm{~kg}^{-1} \mathrm{~s}^{-2}$ the universal gravitational constant, $J_{2}, J_{4}, J_{6}, \ldots$, are the zonal gravitational coefficients and $R_{e}$ is the equatorial radius of the planet. At present, only the first three coefficients $J_{2}, J_{4}, J_{6}$ have been accurately measured for Jupiter but the Juno spacecraft will measure higher-order gravitational coefficients and, hence, provide an accurate spectrum of $J_{n}$ that might contain the gravitational signature of a possible zonal flow in the deep interior of Jupiter. This is because the coefficients $J_{n}$ in (1) consist of two main parts $J_{n}=\left(J_{n}\right)_{0}+\Delta J_{n}:\left(J_{n}\right)_{0}$ produced solely by the effect of rotational distortion and $\Delta J_{n}$ caused by the density anomaly associated with the zonal flow.

Our analysis in this study is different from those of previous studies carried out by Hubbard (1999), Kaspi et al. (2010) and Kong et al. (2013) using approximate numerical methods. Hubbard (1999) computed, in spherical geometry, the density anomaly and the associated values of $\Delta J_{n}$, induced by internal zonal flow, via an approximate spectral method by expanding the density anomaly in terms of spherical harmonics and spherical Bessel functions; Kaspi et al. (2010), also working in spherical geometry, obtained the density anomaly, caused by the zonal winds, through the thermal wind equation that merely represents a diagnostic relation, i.e., it does not have a unique solution and does not require any physical boundary condition; Kong et al. (2013) calculated the wind-induced density anomaly in oblate spheroidal geometry based on a finite element method. This study presents the first analytical solution for the density anomaly and the associated values of $\Delta J_{n}$ 
induced by a zonal flow in a Jupiter-like gaseous planet under the spheroidalshape approximation first proposed by Roberts (1963). The accuracy of the spheroidal-shape approximation is checked by computing both the spheroidal and non-spheroidal solutions, confirming that the difference between them is insignificant.

In what follows we begin by presenting the model and governing equations in $\S 2$; the analytical solution is presented in $\S 3$; an example of its application together with the accuracy of the spheroidal-shape approximation is discussed in $\S 4$; and summary and some remarks are given in $\S 5$.

\section{$2 \quad$ Model and Governing Equations}

Our model assumes that a Jupiter-like gaseous planet with mass $M$ and equatorial radius $R_{e}$ is axially symmetric, isolated and rotating rapidly about the symmetry $z$-axis with an angular velocity $\Omega \hat{z}$, and consists of a compressible barotropic fluid (a polytrope of index unity) whose density $\rho^{*}$ is a function only of pressure $p^{*}=K\left(\rho^{*}\right)^{2}$, where $K$ is a constant (Chandrasekhar, 1969; Hubbard, 1999). It also assumes that the planet is differentially rotating and its angular velocity $\Omega \hat{z}$ is described by

$$
\Omega\left(s^{*}\right) \hat{z}=\left[\Omega_{0}+\epsilon \widehat{\Omega}\left(s^{*}\right)\right] \hat{z},
$$

where $\Omega_{0}$ is a constant, $0<\epsilon \ll 1, \widehat{\Omega}$ is a function of the distance $s^{*}$ from the rotation axis and $\epsilon \widehat{\Omega}\left(s^{*}\right) \hat{\boldsymbol{z}} \times \mathbf{r}^{*}$ represents the zonal flow. We consider the basic angular velocity $\Omega_{0}$ to be sufficiently large that departure from spherical symmetry cannot be treated as a small perturbation. In an inertial frame of reference, the equilibrium equations for the rapidly rotating gaseous planet are

$$
\begin{aligned}
{\left[\left(\Omega_{0}+\epsilon \widehat{\Omega}\right) \hat{\boldsymbol{z}} \times \mathbf{r}^{*}\right] \cdot \nabla\left[\left(\Omega_{0}+\epsilon \widehat{\Omega}\right) \hat{\boldsymbol{z}} \times \mathbf{r}^{*}\right] } & =\frac{-1}{\rho^{*}} \nabla p^{*}-\nabla V_{g}^{*} \\
p * & =K\left(\rho^{*}\right)^{2} \\
\nabla^{2} V_{g}^{*} & =4 \pi G \rho^{*}
\end{aligned}
$$

where $\mathbf{r}^{*}$ is the position vector and $V_{g}^{*}$ is the gravitational potential. Equations (3) - (5) are solved subject to the two boundary conditions

$$
p^{*}=0 \text { and } V_{g}^{*}+V_{c}^{*}=\text { constant }
$$

at the outer bounding surface $\mathcal{S}$ of the planet which is assumed to be an oblate spheroid (Roberts, 1963), and where $V_{c}^{*}$ denotes the centrifugal potential. A recent calculation of the fully self-consistent solution (Kong et al., 2015) shows 
that the outer bounding surface of Jupiter-like planets differs only slightly from an oblate spheroid and, hence, the spheroidal-shape approximation (Roberts, 1963 ) is reasonably accurate for those planets.

Equations (3)-(5) are solved by a perturbation method making use of the expansion

$$
p^{*}=p_{0}^{*}+\epsilon p_{1}^{*}, \quad \rho^{*}=\rho_{0}^{*}+\epsilon \rho_{1}^{*}, \quad V_{g}^{*}=\left(V_{g}^{*}\right)_{0}+\epsilon\left(V_{g}^{*}\right)_{1}
$$

where the leading-order solution $\left(p_{0}^{*}, \rho_{0}^{*}\right)$ represents a hydrostatic state that accounts for the effect of rotational distortion but is unaffected by the zonal flow, and the next-order solution $\left(\epsilon p_{1}^{*}, \epsilon \rho_{1}^{*}\right)$ represents the perturbation arising from the effect of a zonal flow. We concentrate on the density anomaly $\epsilon \rho_{1}^{*}$ that leads to the gravitational signature produced by the zonal flow.

\section{Analytical solution for the density anomaly}

Substituting (7) into (3)-(5), collecting the perturbation variables such as $\rho_{1}^{*}$ at the order of $\epsilon$, combining the resulting equations into a single equation and, then, scaling it with the length scale $R_{e}$ and the density scale $M / R_{e}^{3}$, we obtain a partial differential equation describing the non-dimensional density anomaly $\rho_{1}$,

$$
\left(\frac{2 \pi G R_{e}^{2}}{K}\right) \rho_{1}(\mathbf{r})+\nabla^{2} \rho_{1}(\mathbf{r})=\frac{\Omega_{0}^{2} R_{e}^{5}}{M K}\left[s \frac{\partial \widehat{\Omega}(s)}{\partial s}+2 \widehat{\Omega}(s)\right]
$$

subject to the condition

$$
\rho_{1}=0
$$

at the bounding surface $\mathcal{S}$ of the planet. All the variables without superscript $*$ are dimensionless.

When deriving an analytical solution for (8) in a spheroidal planet, it is mathematically convenient to adopt oblate spheroidal coordinates, $(\xi, \eta, \phi)$, defined by the coordinate transformation with cartesian coordinates $(x, y, z)$

$$
\begin{aligned}
& x=\mathcal{F} \sqrt{\left(1+\xi^{2}\right)\left(1-\eta^{2}\right)} \cos \phi, \\
& y=\mathcal{F} \sqrt{\left(1+\xi^{2}\right)\left(1-\eta^{2}\right)} \sin \phi, \\
& z=\mathcal{F} \xi \eta,
\end{aligned}
$$


where $\xi=$ constant represents a confocal oblate spheroid, $\eta=$ constant describes confocal hyperboloids and $\mathcal{F}$ is the common focal length of an oblate spheroid. It follows that the outer bounding surface $\mathcal{S}$ of the planet, under the spheroidal-shape approximation, is described by

$$
\xi=\sqrt{\frac{1}{\mathcal{E}^{2}}-1} \text { with } 0<\mathcal{E}<1,
$$

where e is the eccentricity of $\mathcal{S}$. With the oblate spheroidal coordinate system, the interior domain $\mathcal{D}$ of the planet is defined by

$$
-1 \leq \eta \leq 1, \quad 0 \leq \xi \leq \sqrt{\frac{1}{\mathcal{E}^{2}}-1}, \quad 0 \leq \phi \leq 2 \pi .
$$

An important application of the solution is to probe the structure of an internal zonal flow via its gravitational signature. In this connection, we introduce a parameterized zonal flow whose velocity $\mathbf{u}$ in the rotating frame of reference is of the form

$$
\mathbf{u}=\epsilon\left(1+\xi^{2}\right)\left(1-\eta^{2}\right) \mathcal{E}^{2} \sin \left[\left(N+\frac{1}{2}\right) \pi \mathrm{e} \sqrt{\left(1+\xi^{2}\right)\left(1-\eta^{2}\right)}\right] \hat{\boldsymbol{\phi}}
$$

where the dimensionless velocity $\mathbf{u}$ is scaled by $\Omega_{0} R_{e}$, and $N$, an integer, reflects the cylindrical radial structure of the zonal flow which is treated as a parameter.

It can be shown that an analytical solution for the inhomogeneous partial differential equation (8) satisfying condition (9) is expressible as

$$
\begin{aligned}
\rho_{1}\left(\xi^{\prime}, \eta^{\prime}\right) & =\frac{2 \pi \mathcal{E}^{4} \Omega^{2} R_{e}^{5}}{M K} \int_{0}^{\xi_{0}} \int_{-1}^{+1}\left(\xi^{2}+\eta^{2}\right) G\left(\xi, \eta ; \xi^{\prime}, \eta^{\prime}\right) \\
& \times \sqrt{\left(1+\xi^{2}\right)\left(1-\eta^{2}\right)}\left\{3 \sin \left[\left(N+\frac{1}{2}\right) \pi \mathcal{E} \sqrt{\left(1+\xi^{2}\right)\left(1-\eta^{2}\right)}\right]\right. \\
& +\left(N+\frac{1}{2}\right) \pi \mathcal{E} \sqrt{\left(1+\xi^{2}\right)\left(1-\eta^{2}\right)} \\
& \left.\times \cos \left[\left(N+\frac{1}{2}\right) \pi \mathcal{E} \sqrt{\left(1+\xi^{2}\right)\left(1-\eta^{2}\right)}\right]\right\} \mathrm{d} \eta \mathrm{d} \xi
\end{aligned}
$$

where

$$
\begin{aligned}
& G\left(\xi, \eta ; \xi^{\prime}, \eta^{\prime}\right)=\left(\frac{G R_{e}^{2}}{2 \pi K}\right)^{1 / 2} \sum_{n=0}^{\infty} S_{0 n}(\eta) S_{0 n}\left(\eta^{\prime}\right) \\
& \quad \times\left[R_{0 n}^{(1)}(i \xi) R_{0 n}^{(2)}\left(i \xi^{\prime}\right)-\frac{R_{0 n}^{(2)}\left(i \xi_{0}\right)}{R_{0 n}^{(1)}\left(i \xi_{0}\right)} R_{0 n}^{(1)}(i \xi) R_{0 n}^{(1)}\left(i \xi^{\prime}\right)\right], \xi<\xi^{\prime}
\end{aligned}
$$




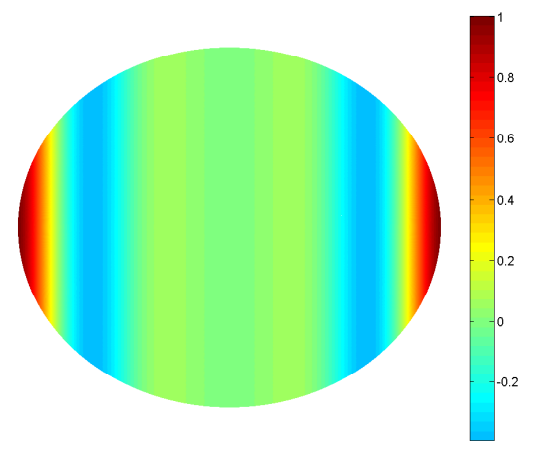

(a)

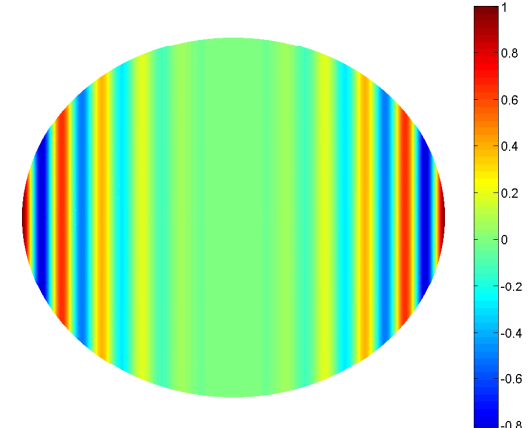

(b)

Fig. 1. Two profiles of the the zonal flow $\mathbf{u}$ in the rotating frame given by (10): (a) a large cylindrical-radial-scale zonal flow with $N=2$ and (b) a small cylindrical-radial-scale zonal flow with $N=10$.

and

$$
\begin{aligned}
& G\left(\xi, \eta ; \xi^{\prime}, \eta^{\prime}\right)=\left(\frac{G R_{e}^{2}}{2 \pi K}\right)^{1 / 2} \sum_{n=0}^{\infty} S_{0 n}(\eta) S_{0 n}\left(\eta^{\prime}\right) \\
& \left.\quad \times\left[R_{0 n}^{(1)}\left(i \xi^{\prime}\right) R_{0 n}^{(2)}(i \xi)-\frac{R_{0 n}^{(2)}\left(i \xi_{0}\right)}{R_{0 n}^{(1)}\left(i \xi_{0}\right)} R_{0 n}^{(1)}\left(i \xi^{\prime}\right) R_{0 n}^{(1)}(i \xi)\right)\right], \xi>\xi^{\prime} .
\end{aligned}
$$

In this solution, $i=\sqrt{-1}, S_{0 n}(\eta)$ denotes the axisymmetric spheroidal angle function, $R_{0 n}^{(1)}(i \xi)$ and $R_{0 n}^{(2)}(i \xi)$ represent two different kinds of axisymmetric spheroidal radial functions (Flammer , 1957). For instance, the spheroidal angle function $S_{0 n}(\eta)$ is a solution of the ordinary differential equation

$$
0=\frac{\mathrm{d}}{\mathrm{d} \eta}\left[\left(1-\eta^{2}\right) \frac{\mathrm{d}}{\mathrm{d} \eta} S_{0 n}(\eta)\right]+\left(\lambda_{0 n}+c^{2} \eta^{2}\right) S_{0 n}(\eta),
$$

which defines an eigenvalue problem in the interval $-1 \leq \eta \leq 1$ with the real but generally non-integer eigenvalues $\lambda_{0 n}$. It is analytical in the sense that the oblate spheroidal wave function is regarded as analytical while the integration in (11) is performed numerically. We refer to the classical monograph (Flammer , 1957) for a detailed discussion of the spheroidal wave functions. Expression (11) represents an analytical solution describing the density anomaly $\rho_{1}$ that is induced by the parameterized zonal flow (10) in a rapidly rotating Jupiter-like gaseous planet.

\section{An example of the analytical solution}

We now apply, as an example, the analytical solution (11) to a generic Jupiterlike planet with the mass $M=1.178009 \times 10^{27} \mathrm{~kg}$, equatorial radius $R_{e}=$ 


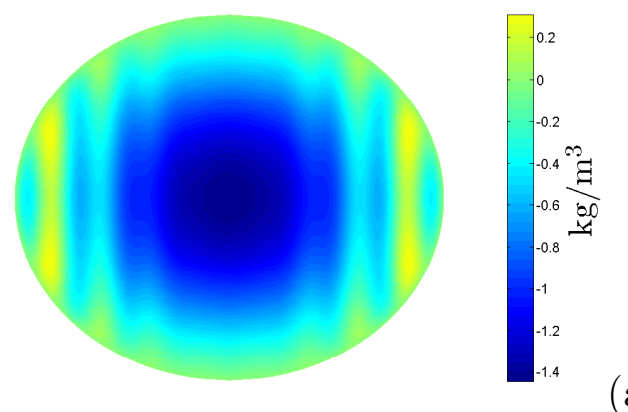

(a)

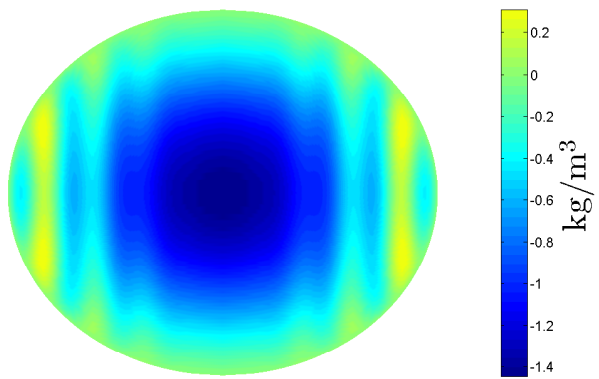

(b)

Fig. 2. The density anomaly $\epsilon \rho_{1}^{*}$ in a meridional plane induced by the zonal flow with the small cylindrical radial scale $N=10$ : (a) $\left(\epsilon \rho_{1}^{*}\right)_{\text {analytic }}$ obtained from the analytical solution (11) and (b) $\left(\epsilon \rho_{1}^{*}\right)_{\text {numeric }}$ computed from the corresponding numerical solution.

$7.565 \times 10^{7} \mathrm{~m}$, and basic angular velocity $\Omega_{0}=2.0 \times 10^{-4} \mathrm{~s}^{-1}$. In this example, $\Omega_{0}$ is slightly larger than Jupiter's $\Omega_{\text {Jupiter }}=1.7585 \times 10^{-4} \mathrm{~s}^{-1}$ so that the effect of rotational distortion is more pronounced and, thus, can be more readily illustrated. Determined by an iterative method, the leading-order solution produces the density $\rho_{0}$, the eccentricity $\mathcal{E}=0.523030$ (corresponding to the ellipticity $e=0.147686)$ and the associated values of $\left(J_{n}\right)_{0}$ along with $K=2.0 \times 10^{5} \mathrm{~Pa} \mathrm{~m}^{6} \mathrm{~kg}^{-2}$. Our focus is, however, on the density anomaly $\rho_{1}$ and the corresponding variation $\Delta J_{n}$ caused by the zonal flow.

Though the zonal winds at the cloud level of giant planets like Jupiter and Saturn exhibit complex structure, we know very little about their relationship to the internal structure of the zonal flow. We therefore consider several different cases for the purpose of illustration. In the first case, the zonal flow is strong and its cylindrical radial scale is large and characterized by a cylindrical radial wavenumber $N=2$ which is depicted in Figure 1(a); in the second case, the differential rotation is also strong but its cylindrical radial scale is small and characterized by a larger wavenumber $N=10$ shown in Figure 1(b). For both cases, the amplitude $\epsilon$ of the zonal flow is taken to be 0.01 , corresponding to a typical speed of the zonal winds $O(100) \mathrm{m} / \mathrm{s}$. As an example, Figure 4(a) shows the density anomaly $\rho_{1}$ in a meridional plane obtained from the analytical solution (11) with $N=10$. In order to validate the analytical solution, we also calculate the corresponding numerical solution using a fully 3-D finite element method (Kong et al., 2013) which is computationally expensive; the numerical solution is displayed in Figure 4(b). It can be seen that there exist no noticeable differences between the analytical $\left(\left(\rho_{1}^{*}\right)_{\text {analytic }}\right)$ and numerical $\left(\left(\rho_{1}^{*}\right)_{\text {numeric }}\right)$ solutions in Figure 4 . Furthermore, the relative error between the two solutions can be measured by

$$
\frac{\left[\iiint_{\mathcal{D}}\left|\left(\rho_{1}^{*}\right)_{\text {analytic }}-\left(\rho_{1}^{*}\right)_{\text {numeric }}\right|^{2} \mathrm{~d} V\right]^{1 / 2}}{\left[\iiint_{\mathcal{D}}\left|\left(\rho_{1}^{*}\right)_{\text {analytic }}\right|^{2} \mathrm{~d} V\right]^{1 / 2}}=6.1 \times 10^{-4},
$$




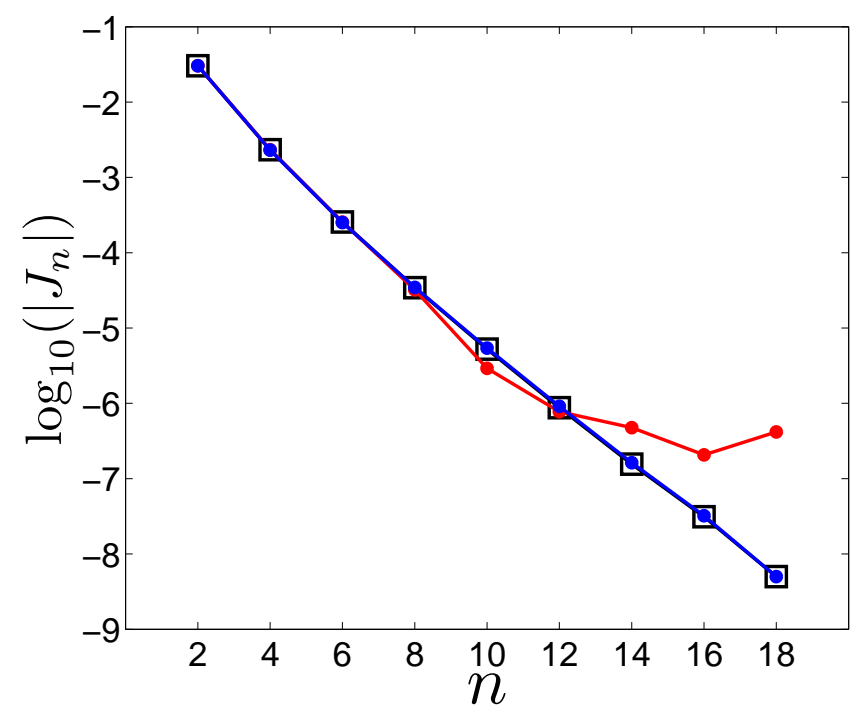

Fig. 3. The zonal gravitational coefficients are shown as function of $n$, where the open squares denote the result of the leading-order solution $\left(J_{n}\right)_{0}$ and the solid circles represent the actual values of $\left(J_{n}\right)_{0}+\Delta J_{n}$. The blue curve shows $\left(J_{n}\right)_{0}+\Delta J_{n}$ for the case with large cylindrical radial scale $N=2$ while the red curve represents $\left(J_{n}\right)_{0}+\Delta J_{n}$ for the case with small cylindrical radial scale $N=10$.

which is reasonable, considering that $\rho_{\text {numeric }}$ is obtained from the highly complicated 3-D numerical analysis.

After obtaining the density anomaly $\rho_{1}$ from either an analytical solution or a numerical solution, we can derive the variation $\Delta J_{n}$ of the gravitational coefficient $\left(J_{n}\right)_{0}$ by performing the following integration

$$
\Delta J_{n}=\frac{-(2 n+1)}{2} \int_{0}^{\pi}\left[\iiint_{\mathcal{D}} \frac{\epsilon \rho_{1}\left(\boldsymbol{r}^{\prime}\right) \mathrm{d}^{3} \boldsymbol{r}^{\prime}}{\left|\boldsymbol{r}-\boldsymbol{r}^{\prime}\right|}\right]_{|\boldsymbol{r}|=1} P_{n}(\cos \theta) \sin \theta \mathrm{d} \theta .
$$

The spectrum of $\Delta J_{n}$ or $\left(J_{n}\right)_{0}+\Delta J_{n}$ reflects the spatial structure of the internal zonal flow. Several values of the zonal gravitational coefficients $\Delta J_{n}$, up to $n=$ 12 , computed from both the analytical and numerical solutions with the same physical parameters and geometry, are listed in Table 1, showing again a good agreement between them. Figure 5 shows the spectrum of the gravitational coefficients $J_{n}=\left(J_{n}\right)_{0}+\Delta J_{n}$, up to $n=18$, for both $N=2$ and $N=10$. When the zonal flow has the large cylindrical radial scale with $N=2$, the variation $\Delta J_{n}$, caused by the zonal flow is always small (up to $n=18$ ) compared to the size of $\left(J_{n}\right)_{0}$ (the open squares in Figure 5) caused by the rotational distortion. In consequence, the spectrum $J_{n}$ is dominated by the leading-order solution $\left(J_{n}\right)_{0}$ (the blue curve in Figure 5): there are no noticeable differences between the spectrum $\left(J_{n}\right)_{0}$ for a rigid-body rotating planet and the spectrum $\left(J_{n}\right)_{0}+$ $\Delta J_{n}$ for a differentially rotating planet. When the zonal flow with the same amplitude has the small cylindrical radial scale with $N=10$, the spectrum $J_{n}$ for $n>10$ is no longer dominated by the leading-order solution $\left(J_{n}\right)_{0}$ (the red 
Table 1

Gravitational zonal coefficients $\Delta J_{n}$ obtained from the analytical solution, denoted as $\left(J_{n}\right)_{\text {analytic }}$, and from the corresponding finite element solution, denoted as $\left(J_{n}\right)_{\text {numeric }}$, for the case with $N=10$. Both the solutions are carried out using the spheroidal-shape approximation.

\begin{tabular}{crr}
$n$ & $\Delta J_{n} \times 10^{6}$ (analytic) & $\Delta J_{n} \times 10^{6}$ (numeric) \\
\hline \hline 2 & & \\
4 & 9.77 & 9.78 \\
6 & -0.38 & -0.38 \\
8 & -3.16 & -3.16 \\
10 & 3.94 & 3.94 \\
12 & -2.96 & -2.96 \\
\hline
\end{tabular}

curve in Figure 5) and the spectrum $\left(J_{n}\right)_{0}$ for a rigid-body rotating planet is characteristically different from that of $\left(J_{n}\right)_{0}+\Delta J_{n}$ for a differentially rotating planet (the red curve in Figure 5). Although the amplitude of the zonal flow is kept exactly the same, the structure of the spectrum of $J_{n}$ can reveal or constrain the structure of an internal zonal flow in rapidly rotating Jupiterlike planets.

An important question is concerned with the accuracy of the spheroidalshape approximation. This question can be firmly answered by comparing the spheroidal analytical solution to the non-spheroidal numerical solution at exactly the same physical parameters. Table 2 shows an example of the zonal gravitational coefficients $\Delta J_{n}$, up to $n=12$, induced by the zonal flow with $N=6$. Two different sets of the coefficients are presented: the analytical values derived using the spheroidal-shape approximation in which the free surface condition is approximately satisfied; and the numerical values obtained with a finite element method in which the outer bounding surface is non-spheroidal and the free surface condition is precisely satisfied. It can be seen that the differences between the analytical (spheroidal) and numerical (non-spheroidal) solutions is insignificant for example,

$$
\frac{\left|\left(\Delta J_{2}\right)_{\text {analytic }}-\left(\Delta J_{2}\right)_{\text {non-spheroidal }}\right|}{\left|\left(\Delta J_{2}\right)_{\text {analytic }}\right|}=0.0001 \%
$$

and

$$
\frac{\left|\left(\Delta J_{12}\right)_{\text {analytic }}-\left(\Delta J_{12}\right)_{\text {non-spheroidal }}\right|}{\left|\left(\Delta J_{12}\right)_{\text {analytic }}\right|}=0.001 \%
$$


Table 2

Gravitational zonal coefficients $\Delta J_{n}$ obtained from the analytical solution using the spheroidal-shape approximation and from the numerical, non-spheroidal solution for the case with $N=6$.

\begin{tabular}{crr}
$n$ & $\Delta J_{n} \times 10^{6}$ (analytic) & $\Delta J_{n} \times 10^{6}$ (non-spheroidal) \\
\hline \hline 2 & & \\
4 & 13.8864 & 13.7458 \\
6 & -5.9181 & -6.1991 \\
8 & 7.6509 & 7.7361 \\
10 & -5.7928 & -5.8477 \\
12 & 2.0076 & 2.0714 \\
\hline
\end{tabular}

In other words, the spheroidal-shape approximation enables analytical progress, thus, provides helpful insight into the problem, and is also highly accurate for Jupiter-like planets.

\section{$5 \quad$ Summary and remarks}

This paper presents the first analytical solution for the density anomaly which is expressed in terms of the spheroidal angle function and the spheroidal radial functions (Flammer , 1957) - induced by a parameterized zonal flow in rapidly rotating Jupiter-like planets. This is also the first time that the spheroidal wave functions - which are much more complicated than the widelyused spherical harmonic functions - are employed to compute the density anomaly induced by the zonal flow in rotating astrophysical gaseous bodies. For the purpose of validation, we also show, by comparing the analytical solution to the corresponding numerical solution based on a finite element method, that an excellent agreement between the analytical and numerical solutions is achieved. Of course, the analytical solution is exact while the numerical solution is not only approximate but also computationally very expensive. We apply the analytical solution to a Jupiter-like gaseous planet, revealing that there exists a relationship between the structure of the zonal flow and the spectrum of the planet's gravitational coefficients. We then reveal that the spheroidal-shape approximation (Roberts, 1963) not only enables analytical progress but also is highly accurate. 


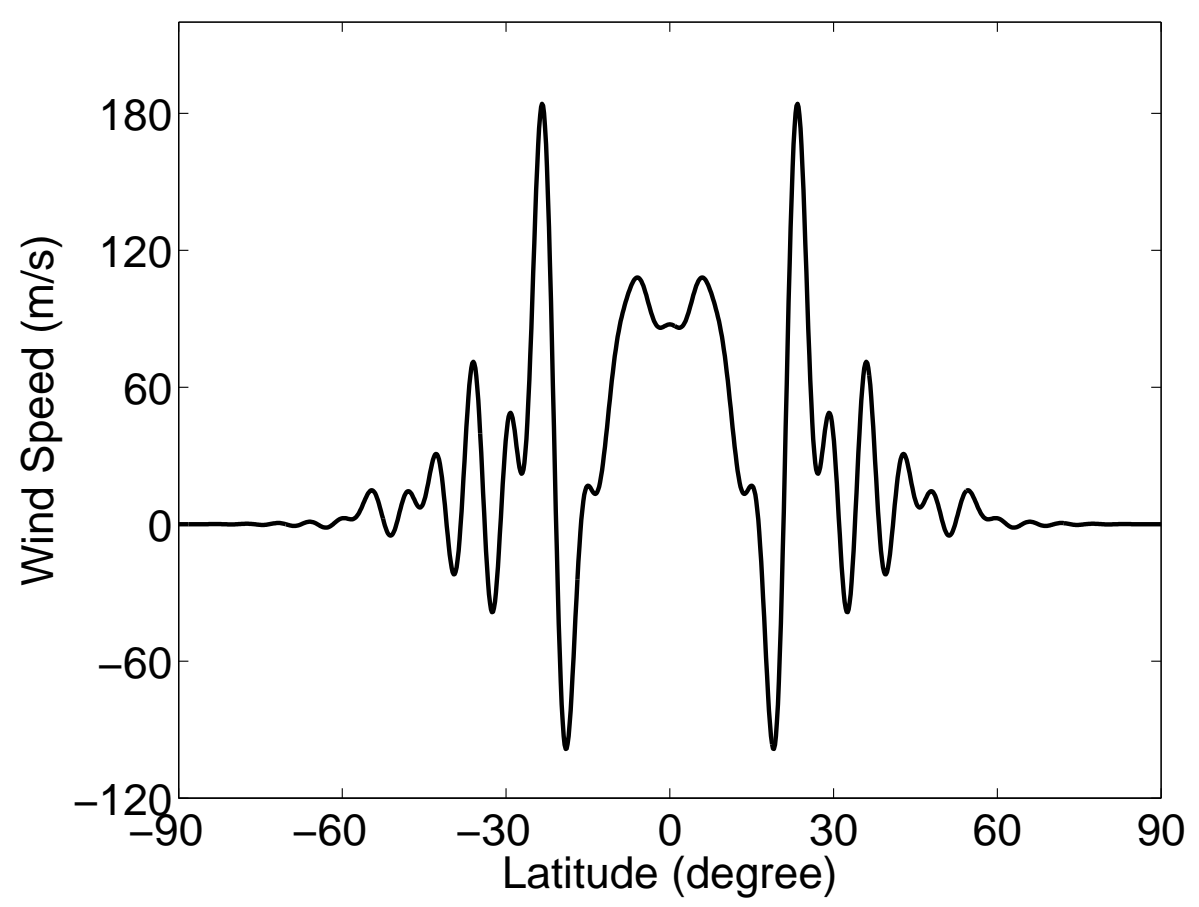

Fig. 4. The profile of $\widehat{\Omega}_{1}$ as a function of the distance $s$ from the rotation axis: the dashed line for $D \rightarrow \infty$ corresponds to the observed Jovian zonal winds extending from the outer surface at all the latitudes to the metallic-molecular interface and the solid line for $D=0.1$ corresponds to the zonal winds confined within the equatorial region between the latitudes $\phi= \pm 25^{\circ}$ with the maximum depth about $10 \% R_{e}$ and containing $0.18 \%$ of the Jovian total mass.

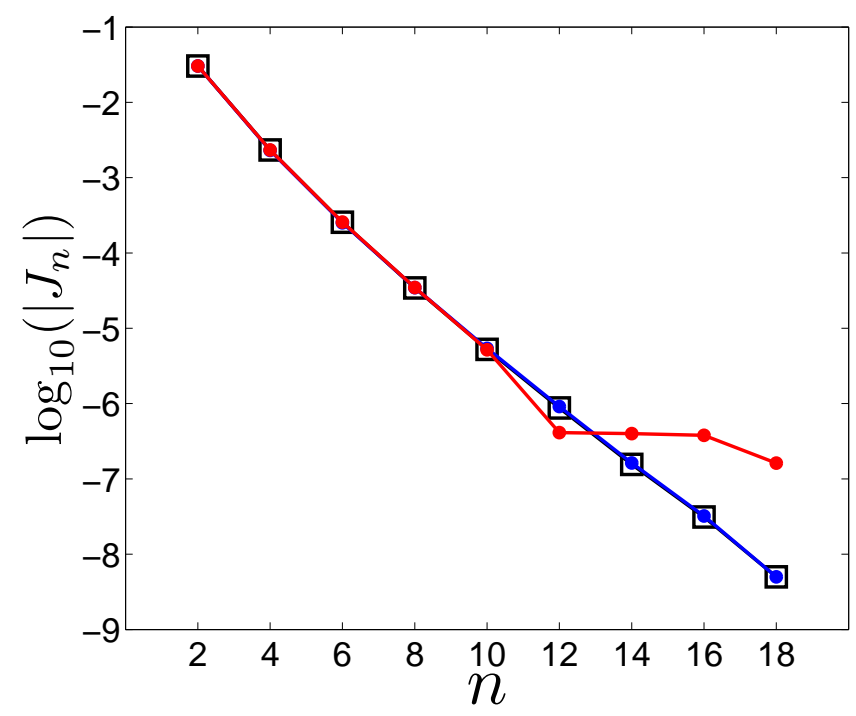

Fig. 5. The zonal gravitational coefficients are shown as function of $n$, where the open squares denote the result of the leading-order solution $\left(J_{n}\right)_{0}$ and the solid circles represent the actual values of $\left(J_{n}\right)_{0}+\Delta J_{n}$. The blue curve shows $\left(J_{n}\right)_{0}+\Delta J_{n}$ for the case with large cylindrical radial scale $N=2$ while the red curve represents $\left(J_{n}\right)_{0}+\Delta J_{n}$ for the case with small cylindrical radial scale $N=10$. 
As an example, we have only applied the analytical solution to a simple profile of the zonal flow given by expression (10). The analytical solution (8) can be readily modified for studying an arbitrary form of the zonal flow in a rapidly rotating gaseous planet of arbitrary eccentricity e. With an accurate spectrum of the zonal gravitational coefficients to be provided by the Juno spacecraft in late 2016 and with an analytical solution that enables straightforward/accurate computation for the spectrum produced by any given profile of a zonal flow, one will be in a position to establish an inverse problem that can constrain the structure of the zonal flow in the deep interior of Jupiter by comparing the observed spectrum to that computed from the analytical solution.

\section{Acknowledgements}

KZ is supported by Leverhulme Trust Research Project Grant RPG-2015-096 and by Macau FDCT grants 039/2013/A2 and 007/2016/A1. The computation made use of the high performance computing resources in the Core Facility for Advanced Research Computing at Shanghai Astronomical Observatory, Chinese Academy of Sciences.

\section{References}

Busse, F. 1976. A simple model of convection in the Jovian atmosphere. Icarus. 29, 255-260.

Chandrasekhar, S., 1969, Ellipsoidal Figures of Equilibrium, Yale University Press, New Haven, CT

Flammer, C. 1957. Spheroidal Wave Functions. Standford University Press, Stanford, California.

Gastine, T., Wicht, J. 2012. Effects of compressibility on driving zonal flow in gas giants. Icarus. 219, 428-442.

Heimpel, M., Aurnou, J.M., Wicht, J. 2005. Simulation of equatorial and high latitude jets on Jupiter in a deep convection model. Nature, 438, 193-196.

Hubbard, W. B., 1999. Gravitational signature of Jupiter's deep zonal flows. Icarus, 137, 357-359.

Jones, C. A. and Kuzanyan, K. M. 2012. Compressible convection in the deep atmospheres of giant planets. Icarus, 204, 227-238.

Kaspi, Y., Hubbard, W. B., Showman, A. P., Flierl, G. R. 2010. Gravitational signature of Jupiter's internal dynamics. Geophys. Res. Lett. 37, L01204.

Kong, D., Liao, X., Zhang, K. and Schubert, G., 2013. Gravitational signature of rotationally distorted Jupiter caused by deep zonal winds. Icarus, 226, $1425-1430$ 
Kong, D., Zhang, K., Schubert, G. 2015. Self-consistent internal structure of a rotating gaseous planet and its comparison with an approximation by oblate spheroidal equidensity surfaces. Physics of the Earth and Planetary Interiors. 249, 43-50.

Roberts, P. H., 1963, On Highly Rotating Polytropes II, Astrophys. J., 138, 809-819

Wisdom,?J.; Hubbard,?W.?B.

Wisdom and Hubbard (2016, Icarus 267, 315-322) "Differential rotation in Jupiter: a comparison of methods". ?This is a concern because the results in Fig. 5 of Wisdom and Hubbard (2016) are nearly identical to the results in Fig. 3 of this manuscript.

Y KaspiJE DavighiE GalantiWB Hubbard There is no mention of Kaspi et al (2016, Icarus 276, 170-181) "The gravitational signature of atmospheric dynamics in giant planets: comparing the thermal wind approach with barotropic potential-surface methods.

TE Dowling

Dowling (1995, Icarus 117, 439-442) "Estimate of Jupiter's deep zonalwind profile from Shoemaker-Levy 9 data and Arnol'd's second stability criterion" 